\title{
Multiple system degeneration with glutamate dehydrogenase deficiency: pathology and biochemistry
}

\author{
S Chokroverty, W Nicklas, D C Miller, J Goldberg, J Choe, C Banner, J Thomas, \\ R Duvoisin
}

\begin{abstract}
The neuropathological findings in a patient with antemortem diagnosis of olivopontocerebellar atrophy (OPCA) and reduced leucocytic glutamate dehydrogenase (GDH) activity included cerebellar cortical degeneration, most marked in the superior vermis, mild atrophy of the pons and the inferior olivary nucleus, marked reduction of anterior horn cells at all levels and gliosis in both lateral columns. GDH activities and their thermolability in "soluble" and "particulate" fractions in the cerebral cortex, cerebellar hemisphere and vermis were not significantly different from the values in two control brains. GDH mRNA in the patient's brain was not altered in size or amount.
\end{abstract}

Departments of Neurology and Pathology, Robert Wood Johnson Medical School, New

Brunswick.

Neurology and

Laboratory Service,

VA Medical Center,

Lyons, New Jersey.

National Institute of

Neurological Diseases

and Stroke, Bethesda,

Maryland, United

States

S Chokroverty

W Nicklas

D C Miller*

J Goldberg

J Choe

C Banner

J Thomas

R Duvoisin

Correspondence to:

Dr Chokroverty,

PO Box 308, Lyons,

NJ 07939, United States.

^Present address:

Department of Pathology

(Neuropathology), New

York University Medical

Center, 550 First Avenue,

New York, NY 10016,

United States.

Received 16 August 1989 and

in final revised form

Accepted 20 March 1990
Initial reports of an association between low glutamate dehydrogenase (GDH) activity in patients with olivopontocerebellar atrophy (OPCA $)^{1-10}$ have not been confirmed by more recent clinical studies. ${ }^{11} 12$ Moreover, several patients with OPCA and low leucocyte GDH activity have been found at necropsy to have distinctive pathologies. Thus GDH deficiency could not be correlated with a specific morbid entity. The first necropsy study of a patient with GDH deficiency and OPCA showed typical findings of OPCA with diffuse lipofuscinosis. ${ }^{3}$ A second patient had neuronal intranuclear hyaline inclusion disease. ${ }^{13} \mathrm{~A}$ third ${ }^{14}$ appears to have had the DejerineThomas type ${ }^{15}$ of sporadic OPCA. We report the morphological and biochemical findings in a fourth patient with multiple system neuronal degeneration resembling OPCA with marked amyotrophy who had low leucocyte GDH activity. The clinical features of this patient were previously described briefly by Duvoisin et $a l^{2}$ (case 4) and the neurophysiological findings by Chokroverty et $\mathrm{al}^{4}$ (case 3). We now present the necropsy findings including morphological observations and biochemical studies.

\section{Case report}

The illness first appeared with unsteadiness of gait at the age of 49. Examination at the age of 65 revealed mild diffuse weakness of the legs, spasticity, hyperactive muscle stretch reflexes and extensor plantar responses. Sensation and cerebellar functions were normal. Subsequently, speech became slurred and gait was more unsteady requiring a walking stick. His family history was unremarkable. There was no history of heavy alcohol ingestion. The disorder gradually progressed and on examination at the age of 69 the gait was wide-based with frequent small steps. He had mild facial hypomimia and scanning dysarthria. Other findings included mild weakness of the left upper extremity, mild weakness and spasticity in the legs, mild intention tremor in the upper extremities and hyperactive muscle stretch reflexes. Sensation was normal.

Despite rehabilitation therapy he became progressively worse, with increasing weakness and muscle atrophy in all four limbs and dysphagia. At the age of 73 , he had decreased gag reflex bilaterally, moderate weakness of neck flexors and extensors, marked weakness and atrophy in all four limbs, greater proximally with muscle wasting and widespread fasciculations. Muscle tone was decreased in the upper limbs but slightly increased in the lower extremities. Muscle stretch reflexes were absent in the upper limbs and brisk in the legs. Sensation remained normal. The patient was confined to a wheelchair. His condition continued to deteriorate. Multiple episodes of respiratory infection culminated in death from respiratory failure 24 years after the onset of the illness.

Laboratory examination revealed normal cerebrospinal fluid findings. CT scan showed mild cerebral but no cerebellar atrophy. Leucocyte preparations showed reduced GDH activity. Leucocyte GDH was normal in his daughter, aged 33. Electromyographic findings in all four limbs were consistent with diffuse progressive anterior horn cell dysfunction. Nerve conduction studies and an EEG were normal except for reduced or absent sural nerve potentials.

\section{NECROPSY FINDINGS}

Gross examination Necropsy was performed $11 \frac{3}{4}$ hours postmortem. The brain weighed 
Figure Whole mount of midline sagittal-plane section of cerebellar vermis. The folia particularly in the anterior-superior portion (right side of picture) are narrower than normal and have patchy pallor of myelin staining. $S U P=$ Superior. $A N T=$ Anterior. Luxol Fast Blue, haematoxylin and eosin; $3.5 \times$

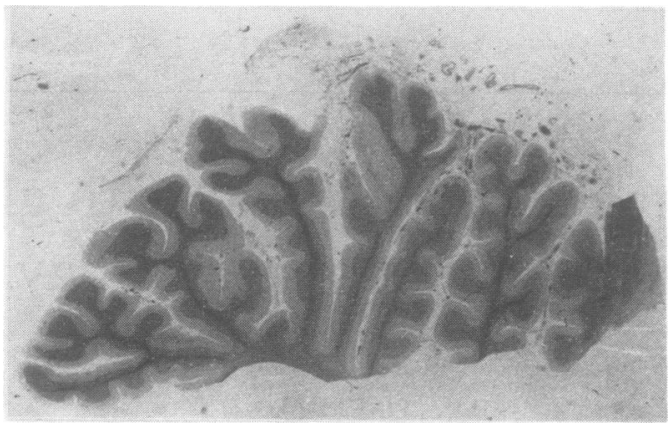

$1230 \mathrm{~g}$. The brain was divided sagitally, including the brainstem and cerebellum, the right half was frozen at $-70^{\circ} \mathrm{C}$. Neuropathological examination of the left half, following formalin fixation, revealed pontine atrophy. The frontal and temporal horns of the lateral ventricle were mildly dilated. On transverse sections of the brainstem and cerebellum the pontine atrophy was principally tegmental. Some of the crossing fibres in the basis pontis, and the VIIIth nerve fibres were thinner than normal. In contrast the midbrain showed no abnormality. The medullary pyramid and inferior olivary nucleus were slightly smaller than normal. The medial cerebellar surface showed moderate atrophy of the superior vermis (fig). The cerebellar hemispheric white matter was also normal.

Microscopic examination A few neuritic ("senile") plaques were seen in the cerebral cortex. The caudate, putamen, thalamus, and substantia innominata were normal. The outer segment of the globus pallidus was gliotic and had lost myelin. The substantia nigra had a normal density of pigmented neurons. The central and lateral portions of the pontine tegmentum were gliotic. Pontine crossing fibres, while thin, were well-myelinated. There was neuronal loss in the nucleus of the tractus solitarius, and nucleus ambiguus accompanied by axonal spheroids and gliosis. The inferior olivary nucleus was thin but not gliotic. The medullary pyramid appeared normal.

The lateral cerebellar cortex had a mild deficit of Purkinje cells with a minimal Bergmann gliosis. Sections from the superior vermis showed marked loss of Purkinje cells, some loss of cells from the granular layer, and a more prominent Bergmann gliosis. The cerebellar deep white matter and the dentate nucleus showed no histological changes.

Mild to moderate gliosis was seen in the lateral columns of the spinal cord, minimally in the upper thoracic cord but progressively more pronounced at lower levels. Anterior horn cells were reduced in number at all levels. The surrounding tissue was gliotic, contained microglia, and some debris-filled macrophages. Some of the remaining motor neurons were rounded, shrunken, or shrivelled, and a few were chromatolytic.

Formalin-fixed, paraffin embedded muscles (deltoid and gastrocnemius) showed typical neurogenic atrophy. Dorsal root ganglia were normal.

\section{BIOCHEMICAL STUDIES}

Method Leucocytes were prepared from patients and control subjects by a modification of previously published methods. ${ }^{2}$ The procedure outlined by Colon et al ${ }^{16}$ was followed in assessing thermolability of soluble and particulate fractions. Aliquots of whole homogenates as well as soluble and particulate fractions were assayed for GDH activity as previously described. ${ }^{2}$

Ribonucleic acid (RNA) from the frontal cortex, cerebellar cortex, and neostriatum was extracted by the procedure of Chirgwin et al. ${ }^{17}$ The results were expressed as a ratio of $\mathrm{GDH}$ mRNA to GAD mRNA. ${ }^{18}{ }^{19}$ Since GDH and GAD mRNAs are of similar size $(3.7 \mathrm{~kb})$, the amount of nonspecific degradation should be similar. GDH/GAD mRNA ratio in the control frontal cortex was taken as unity.

Results The average leucocyte GDH activity in our patient was 0.61 (SD 0.08) units. The control values derived from 26 non-diseased donors was 1.24 (SD 0.34 ) units $^{12}$ and in a concurrent series of 20 multiple system atrophy cases, 1.00 (SD 0.29) units. Low GDH in our patient was persistent and reproducible on assays performed on leucocyte preparations at different times; the patient's total and differential WBC count on those days remained normal.

GDH activity was expressed in $\mu \mathrm{mol} \mathrm{NADH}$ oxidised $/ \mathrm{hr} / \mathrm{mg}$ protein assayed at $35^{\circ} \mathrm{C}$ and the results are shown as mean (SD). GDH activity was not grossly different in the cerebellar hemisphere [Patient: 16.2(0.4); Control 1: 15.1 $(0 \cdot 3)$; Control 2: $14 \cdot 1(0 \cdot 1)$ ], cerebellar vermis [Patient: 12.2 (0.3); Control 1: $11.8(0.8)$; Control 2: $12.5(0 \cdot 8)$ ], cerebral cortex [Patient: 17.7 (2.9); Control 1: 15.2 (4.1); Control 2: $14 \cdot 1$ $(0 \cdot 5)$, basal ganglia [Patient: $17 \cdot 1(1 \cdot 1)$; Control 1: 18.9 (4.5); Control 2: $13.5(2.4)$ ] and inferior olive [Patient: $28 \cdot 1(0 \cdot 2)$; Control 1: $27 \cdot 5(1 \cdot 0)$; Control 2: $29 \cdot 0(1 \cdot 4)$ ] of the patient from values measured in two control brains. The kinetics of ADP stimulation were also not significantly different between the patient and the two controls.

There were no differences between the patient and the two controls in the thermolability of GDH in "soluble" and "particulate" fractions in whole homogenates and cerebellar hemisphere, cerebellar vermis or frontal cortex preparations. These studies were done by incubating the fractions at $45^{\circ} \mathrm{C}$ in a buffer

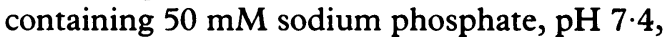
to mimic the conditions used by others. ${ }^{16}$ To study the effects of other salts on thermolability, "particulate" and "soluble" fractions were heat treated in two other buffer systems, $100 \mathrm{mM}$ potassium phosphate and $50 \mathrm{mM}$ tris chloride, both at $\mathrm{pH} 7 \cdot 4$. No differences were seen in any condition between the patient and the two controls.

GDH mRNA was not altered in size in the brain regions including areas showing little or no gliotic response in the patient nor was there any evidence that GDH mRNA was reduced in amount. On the contrary, the ratio of GDH mRNA to GAD mRNA was increased twofold in each region. Another predominantly 
neuronal mRNA, glutaminase mRNA, was reduced in the patient compared to mRNA for GDH.

\section{Discussion}

The initial clinical picture in this patient and the necropsy findings of superior vermis and the anterior lobe atrophy in the cerebellum resemble those described by Marie et al. ${ }^{20}$ This type of clinical-pathological picture has also been described in patients with a restricted form of alcoholic cerebellar degeneration, ${ }^{21}$ but the severe amyotrophy with anterior horn cell loss in the later stages of the illness is difficult to ascribe to alcoholism. The patient and the family members denied any history of moderate to heavy alcohol ingestion in the past. From a morphological perspective this case may best be classified as a form of multiple system atrophy. Our patient differs from that briefly described by Plaitakis and $\mathrm{Smith}^{14}$ in the severe motor neuron involvement.

Our patient repeatedly showed low leucocytic GDH activity, however, GDH activity in both the "soluble" and "particulate" fractions of homogenates from different areas of brains at necropsy showed no significant difference from the values found in our two controls. Moreover, GDH mRNA in the affected brain regions in our patients did not alter in size or amount. It should be noted that the lack of change in GDH mRNA was observed even in brain areas showing little or no gliotic response, thereby obviating a contribution from increased glial GDH.

Grossman et al $^{22}$ found no significant reduction in GDH activities in brain samples at necropsy (cerebellar and cerebral cortex) in four autosomal dominant OPCA patients compared to five controls; and Rosenberg and Banner $^{23}$ found normal GDH activity in cerebellar homogenates from their patient with autosomal dominant OPCA. Thus no abnormality of GDH can be implicated in the pathogenesis of neuronal degeneration in our patient. There appears to be no correlation between leucocytic GDH and brain GDH activity. We could not confirm the presence of $\mathrm{GDH}$ isozymes $^{24}$ distinguished by different thermolabilities. The reason for low leucocytic GDH remains unclear. Aubby et $a l^{11}$ have suggested multiple factors relating to age, physical disability and medication.

In conclusion, the morphological alterations in the central nervous system of a patient clinically identified as a case of OPCA associated with low leucocyte GDH activity differed significantly from the findings noted in the previously reported cases of OPCA with GDH deficiencies studied postmortem. Further, brain GDH activity was not reduced and brain GDH mRNA was normal. Peripheral
GDH "deficiency" does not appear to indicate brain GDH deficiency reliably and may be found in various distinct morbid entities. Further studies in search of aetiological factors in OPCA should focus on molecular genetics rather than on peripheral enzymes or protein abnormalities.

1 Plaitakis A, Nicklas WJ, Desnick RJ. Glutamate dehydrogenase deficiency in 3 patients with spinocerebellar syndrome. Ann Neurol 1980;7:297-303.

2 Duvoisin RC, Chokroverty S, Lepore F, Nicklas W. Glutamate dehydrogenase deficiency in patients with olivopontocerebellar atrophy. Neurology 1983;33:1322-6.

3 Chokroverty S, Khedekar R, Darby B, et al. Pathology of olivopontocerebellar atrophy with glutamate dehydrogenase deficiency. Neurology 1984;34:1451-5.

4 Chokroverty S, Duvoisin RC, Sachdeo R, et al. Neurophysiologic study of olivopontocerebellar atrophy with or without glutamate dehydrogenase deficiency. Neurology 1985;35:652-9.

5 Yamaguchi I, Hayashi $\mathrm{K}$, Murakami $\mathrm{H}$, et al. Glutamate dehydrogenase deficiency in spinocerebellar degeneration. Neurochem Res 1982;7:627-36.

6 Duvoisin RC, Chokroverty S. Clinical expression of glutamate dehydrogenase deficiency. In: Duvoisin RC Plaitakis A, eds. The olivopontocerebellar atrophies, Adv Neurol, New York: Raven Press 1984;40:267-79.

7 Konagaya Y, Konayaga M, Mano Y, Takayanagi T. Glutamate dehydrogenase and its isoenzyme activity in Glutamate dehydrogenase and its isoenzyme activity in
olivopontocerebellar atrophy. $J$ Neurol Sci 1986;72:231-6.

8 Sorbi S, Tonini, S, Giannini E, et al. Abnormal platelet glutamate dehydrogenase activity and activation in dominant and non-dominant olivopontocerebellar atrophy. Ann Neurol 1986;19:239-45.

9 Finocchiaro G, Taroni F, DiDonato S. Glutamate dehydrogenase in olivopontocerebellar atrophies: Leucocytes, fibroblasts, and muscle mitochondria. Neurology 1986;36:550-3.

10 Kajiyama K, Veno S, Tasumi T, et al. Decreased glutamate dehydrogenase protein in spinocerebellar degeneration. J Neurol Neurosurg Psychiatry 1988;51:1078-80.

11 Aubby D, Saggu HK, Jenner P, et al. Leucocyte glutamate dehydrogenase activity in patients with degenerative neurological disorders. I Neurol Neurosurg Psychiatry neurological disor

12 Duvoisin RC, Nicklas WJ, Ritchie V, et al. Low leucocyte glutamate dehydrogenase activity does not correlate with a particular type of multiple system atrophy. $J$ Neuro Neurosurg Psychiatry 1988;51:1508-11.

13 Parker JC, Dyer ML, Paulsen WA. Neuronal intranuclear hyaline inclusion disease associated with premature coronary atherosclerosis. J Clin Neuro-Ophthalmol 1988;7:244-9.

14 Plaitakis A, Smith J. Biochemical and morphological changes of the brain in a patient dying of GDH-deficient olivopontocerebellar atrophy. Ann Neurol 1986;20:152-3.

15 Dejerine J, Thomas A. L'atrophine olivo-pontocerebelleuse. Nouv lcon de laq salpet 1900;13:330-70.

16 Colon AD, Plaitakis A, Perakis A, et al. Purification and characterization of a soluble and a particulate glutamate dehydrogenase from rat brain. $J$ Neurochem 1986; 46(6):1811-9.

17 Chirgwin JM, Przybyla AE, MacDonald RJ, Rutter WJ. Isolation of biologically active ribonucleic acid from sources enriched in ribonuclease. Biochemistry 1979; 18:5294-9.

18 Kaufman DL, McGinnis JF, Krieger NR, Tobin AJ. Brain glutamate decarboxylase cloned in gt-11: Fusion protein produces gamma-aminobutyric acid. Science 1986; 232:1138-40.

19 Banner C, Silverman S, Thomas JW, et al. Isolation of a human brain cDNA for glutamate dehydrogenase. $J$ Neurochem 1987;49(1):246-52.

20 Marie P, Foix C, Alajouanine T. De l'atrophie cerebelleuse tardive a predominance corticale. Rev Neurol 1922, tardive a predominan

21 Victor M, Adams RD, Mancall EL. A restricted form of cerebellar cortical degeneration occurring in alcoholic cerebellar cortical degeneration occurring in
patients. Arch Neurol (Chic) 1959;1:579-688.

22 Grossman A, Rosenberg RN, Warmoth L. Glutamate and malate dehydrogenase activities in Joseph Disease and olivopontocerebellar atrophy. Neurology 1987;37:106-11.

23 Rosenberg R, Banner C. Normal cerebellar glutamate dehydrogenase protein in spinocerebellar degeneration. $J$ Neurol Neurosurg Psychiatry 1989;52:666-8.

24 Plaitakis A, Berl S, Yahr MD. Neurological disorders associated with deficiency of glutamate dehydrogenase. Ann Neurol 1984;15:144-53. 